\title{
The Modal Status of Contextually A Priori Arithmetical Truths
}

\section{Pantsar, Markus}

Springer

2016

Pantsar, M 2016 , The Modal Status of Contextually A Priori Arithmetical Truths . in F Boccuni \& A Sereni (eds), Objectivity, Realism, and Proof : FilMat Studies in the Philosophy of Mathematics . Boston Studies in the Philosophy and History of Science, no. 318 , Springer , pp. 67-79. https://doi.org/10.1007/978-3-319-31644-4_5

http://hdl.handle.net/10138/325185

https://doi.org/10.1007/978-3-319-31644-4_5

acceptedVersion

Downloaded from Helda, University of Helsinki institutional repository.

This is an electronic reprint of the original article.

This reprint may differ from the original in pagination and typographic detail.

Please cite the original version. 


\title{
The modal status of contextually a priori arithmetical truths
}

\author{
May 18, 2016
}

\begin{abstract}
In Pantsar (2014), an outline for an empirically feasible epistemological theory of arithmetic is presented. According to that theory, arithmetical knowledge is based on biological primitives but in the resulting empirical context develops an essentially a priori character. Such contextual a priori theory of arithmetical knowledge can explain two of the three characteristics that are usually associated with mathematical knowledge: that it appears to be a priori and objective. In this paper it is argued that it can also explain the third one: why arithmetical knowledge appears to be necessary. A Kripkean analysis of necessity is used as an example to show that a proper analysis of the relevant possible worlds can explain arithmetical necessity in a sufficiently strong form.
\end{abstract}

\section{Introduction}

At first sight, arithmetical truths appear to have two characteristics that an epistemological theory should be able to explain: they seem to be both objective and necessary. Whether or not this is the case under deeper philosophical analysis, it can hardly be denied that the impression is strong. Although there are obviously exceptions, generally mathematicians, philosophers and laymen all find it hard to believe that, say, " $2+1=3$ " is merely some contract we have agreed upon. They may disagree on what numbers are, or what it means for a truth to be objective, but in some relevant sense they nevertheless regard $2+1$ $=3$ as an objective truth about numbers. But if it indeed is an objective truth, it would also seem to be a necessary truth. If someone is not ready to accept that " $2+1=3$ " is merely a convention, she is not likely to accept that it could have been the case that $2+1=3$ was not true.

In addition to those two impressions, it is common to have the impression that arithmetical knowledge is a priori. ${ }^{1}$ It could be said that these three char-

\footnotetext{
${ }^{1}$ It is not possible here to enter the discussion of what - if anything - constitutes a priori knowledge. In this paper, I will accept the possibility of a priori knowledge and follow the classical understanding that it is knowledge that can be gained in an essentially non-empirical manner.
} 
acteristics form the traditional Kantian (1781) understanding of the nature of arithmetical knowledge. ${ }^{2}$ While this understanding is widely accepted, it has also been challenged from many directions. Conventionalists like Wittgenstein (1956), for example, deny the objectivity of arithmetical truths, while empiricists like Kitcher (1983) reject the a priori nature.

However, there is something potentially troubling in such challenges if they simply reject the impressions that we should be able to explain. The fictionalist Field (1980), for example, argues that the strong impression we have of the truth of certain mathematical theories is mistaken:

What makes the mathematical theories we accept better than these alternatives to them is not that they are true [...] but rather that they are more useful. [...] Thus mathematics is in a sense empirical but only in the rather Pickwickian sense that it is an empirical question as to which mathematical theory is useful. (Field (1980), p. 15. Italics in the original)

But surely we cannot be satisfied to simply leave the matter there. In an account like Field's, the usefulness of some mathematical theories over their alternatives would seem to be one of the key philosophical questions. And just like we must ask why certain theories are useful while others are not, we must ask why the traditional image of arithmetic has been so appealing to mathematicians and philosophers - as well as other scientists and laymen. What is it about arithmetic that makes so many see it as an a priori pursuit of objective and necessary truths? Certainly the reason does not seem to be lack of knowledge about arithmetic, since so many experts subscribe to that view. In philosophy we may argue that arithmetic is neither a priori, objective nor necessary, but even in rejecting all those characteristics we cannot escape the question why it intuitively seems to us to have these characteristics. Ultimately, any epistemological theory of arithmetic should be able to deal with this problem.

In this paper I will study these questions with regard to the contextual a priori theory of epistemology arithmetic proposed in Pantsar (2014). In that account, arithmetical knowledge is thought to be empirically constrained by biological primitives that we have as infants and share with many animals. However, in that empirical context arithmetic is thought to be essentially a priori. I will first show that the appearances of a priori and objectiveness follow directly from that theory. The situation is more difficult, however, when it comes to necessity. Since sufficiently developed biological organisms do not develop in all possible worlds, the conception of necessary truths as being true in all possible worlds seems like a bad fit with the contextual a priori account. I will argue, however, that by limiting ourselves to the relevant set of possible worlds - i.e., ones with sufficiently developed organisms - we get a strong enough conception of necessity. I will argue that this is consistent with a Kripke-esque theory of

\footnotetext{
${ }^{2}$ For Kant, there was of course also the important distinction between synthetic and analytic knowledge. In this paper that distinction, namely the possible differences between arithmetical knowledge being analytic a priori or synthetic a priori, will not be considered.
} 
designation, when qualified for the relevant context of arithmetic. However, the approach is not limited to any particular semantic theory, as it only involves considering necessity in the proper context of arithmetic understood as an ability arising from our cognitive architecture.

\section{Contextual a priori}

In Pantsar (2014), I have proposed an empirically feasible approach to the epistemology of arithmetic. The purpose of the paper is to study what kind of epistemological theory is required if the recent results in neurobiology, cognitive science and psychology are accepted as giving insight to the origins and nature of arithmetical knowledge. Those results suggest that arithmetical knowledge is built on biological primitives that we already possess as infants and share with many nonhuman animals. In short, the brain is structured so that there is a natural tendency to process observations in terms of discrete quantities. This can be seen in subitizing, i.e. the ability to determine the quantity of objects in one's field of vision without counting, as well as in the capacity to estimate quantities and keep them in the working memory. ${ }^{3}$

This primitive ability, what I call proto-arithmetic, should not be confused with developed arithmetical thinking. The proto-arithmetical abilities quickly lose their accuracy as the numerosities become larger, and the quantities can be properly described as discrete only for the numerosities from one to four or five. ${ }^{4}$ However, it is argued that this primitive ability to deal with numerosities works as the basis on which we develop our arithmetic, in a process in which the development of language plays a key role. The central idea is that the proto-arithmetical treatment of numerosities develops into actual arithmetic once explicit rules and number words or symbols are introduced. In developing arithmetical ability, one key stage is understanding some form of successor operation. The proto-arithmetical cognitive system enables understanding that numerals "one", "two", "three", and "four" refer to different numerosities that form a succession. In learning to count, we generalize on this process, that is,

\footnotetext{
${ }^{3}$ The empirical details and references can be found in Pantsar (2014). For an introduction to the empirical studies, see Dehaene (2011) and Dehaene \& Brannon (eds.) (2011). In the latter, the paper by Nieder (2011) is particularly recommended as a good window to the state of the art in the empirical research of proto-arithmetical cognition.

${ }^{4}$ Hence at the level of proto-arithmetic, it is better to talk of numerosities rather than numbers. This distinction is too rarely made in the empirical literature. Unfortunately, it is also common in the empirical literature to postulate needlessly strong abilities to the test subjects. For example, in one of the most famous papers, Wynn (1992), it is shown that infants react to unnatural numerosities in experimental settings. When they see one and one dolls put behind a screen but only one doll when the screen is removed, they are surprised. This was an important result and further experiments have shown the proto-arithmetical ability involved in the process to be a particularly important one. The unnatural numerosity actually surprises infants more than changes in the position, sizes, or even the character of the doll. Nevertheless, when Wynn called her paper "Addition and subtraction by human infants", she seems to postulate needlessly strong ability to her test subject. The infants could simply have the ability to hold one numerosity in their working memory and be surprised when the observations don't match that.
} 
we understand that the step from "three" to "four" is no different from the step from "four" to "five, etc. (Carey \& Sarnecka 2006, Spelke 2011, Pantsar 2014).

The final step of this development is formal arithmetic, which has traditionally been the subject of philosophy of arithmetic. But to understand arithmetic only as dealing with formal systems seems needlessly limiting, especially epistemologically. A key feature of my account is to see arithmetical knowledge as a subject involving different levels of ability with numerosities. Only then, he claims, can we fully understand the nature of arithmetical knowledge.

Thus characterized, arithmetical knowledge has minimal ontological and epistemological requirements. No mind-independent existence of arithmetical objects is assumed and no special epistemic faculty for arithmetical knowledge is needed - but neither is either of those denied. My main claim is that such a development from the proto-arithmetical ability based on biological primitives to the language-based arithmetical thinking is enough to explain the nature of arithmetical knowledge - including the impression we have of it as being a priori, objective and necessary.

In my outline for an epistemological theory for arithmetic, I characterize arithmetical knowledge thus understood as contextually a priori. The main idea behind that characterization is that arithmetical knowledge is indeed essentially a priori in character, but only in a context set by empirical facts. We do not accept the statement " $2+1=3$ " because it can be derived from a set of arithmetical axioms which are knowable a priori, or because we have a special non-sensory arithmetical intuition. We accept it initially because by the time we have learned the necessary number words or symbols, we already have a vast experience of processing observations in terms of quantities. Our brain structure forces upon us the notion that one object added to two objects make three objects. From the point of view of a child learning about addition, she initially accepts " $2+1=3$ " because it counts as an empirical fact - it corresponds to how she has experienced the world all her life.

However, after this empirical context is set, arithmetic does not work like empirical sciences. It has its own methodology of proof procedures (both formal and informal) that does not rely on empirical corroboration. While the roots of arithmetic are in empirical aspects, in that empirical context arithmetical knowledge is essentially a priori. ${ }^{5}$

It is important to note that in the contextual a priori account, the empir-

\footnotetext{
${ }^{5}$ My epistemological theory is related to, but different from, other accounts emphasizing the empirical foundations of arithmetic, e.g. Kitcher (1983), Lakoff \& Núnez (2000) and Jenkins (2008). In all the accounts perhaps the key question is how we can combine the position that arithmetical concepts are empirically grounded with the the image of arithmetic as an a priori pursuit. The solutions range from rejecting the apriority (Kitcher) to accepting that arithmetical knowledge is essentially both empirical and a priori (Jenkins). One key feature of my account is to soften the notion of a priori applicable to arithmetic. However, it should be noted that the account of contextual a priori is fundamentally different from the ones by Kuhn (1993) and Putnam (1976). For me, arithmetic is not simply a paradigm that is a priori in one context but could be overthrown when that context changes. Rather, arithmetical knowledge is based on biological primitives and as such it is based on an effectively inevitable way of experiencing the world.
} 
ical aspects do not play a merely enabling role for arithmetical knowledge. It is universally accepted that empirical methods, like manipulating collections of pebbles, help us learn arithmetic. But Frege (1884) famously distinguished those psychological processes (the context of discovery) from what makes arithmetical propositions true (the context of justification). In the beginning of Begriffsschrift (1879) he presents this distinction:

we can inquire, on the one hand, how we have gradually arrived at a given proposition and, on the other, how it is finally to be most securely grounded. The first question may have to be answered differently for different persons; the second is more definite, and the answer to it is connected with the inner nature of the proposition considered.

When arguing that arithmetical knowledge is based on empirical aspects, am I mixing up the two contexts? Clearly this is not the case. While I take empirical aspects to be at the basis of arithmetical knowledge, there is an obvious difference in how an indiviual arrives at a given proposition (the discovery) and what the content of that proposition is. In studying the empirical roots or arithmetic, I am concerned with the inner nature of the proposition - I only claim that this inner nature cannot be fully explained in philosophy without tracking it back to its cognitive roots.

In the contextual a priori account, when we establish arithmetical truths, we do not engage in an empirical pursuit. The justification comes, just as for Frege, from an essentially a priori process. The contextual a priori account does not make arithmetic an empirical science that allows psychological processes to take over logical analysis as the justification of arithmetical statements. For all practical intents and purposes, arithmetic in the contextual a priori account is the same a priori discipline as it was for Frege.

The difference comes when we consider why arithmetical truths are what they are. This has traditionally been perhaps the most important question in the philosophy of mathematics and the answers have ranged from a Platonic world of mathematical objects to mathematics being an empirical science. The contextual a priori account is one answer to that question. It states that arithmetical truths are ultimately constrained by the proto-arithmetical structure of our observations, determined by our cognitive architecture. It is that context that gives us the conception that there are such things as discrete quantities and they form a succession, and it is in that context that we justify arithmetical truths with logical methods.

Thus characterized, the contextual a priori account gives a direct explanation for one of the three impressions we have about arithmetical knowledge: that it is a priori in character. Once the empirical context is set - which happens early on in our lives - arithmetic is essentially a priori.

In addition to apriority, the account also gives a satisfactory explanation for the apparent objectivity of arithmetical truths. If arithmetic is based in such a way on biological primitives, arithmetical knowledge is based on an effectively inevitable way of experiencing the world. I argue that this gives arithmetical 
knowledge a sufficiently strong form of objectivity. We cannot help experiencing the world in terms of quantities, and this tendency is the basis of arithmetic. Perhaps we would like to call this "maximal intersubjectivity" rather than objectivity, but the important point is that arithmetical knowledge does not succumb to the conventionalist threat. Arithmetical truths - unlike, say, the rules of chess - are not only a matter of commonly agreed contract, and as such, we would expect them to appear objective to us.

In this way, the account for arithmetical knowledge in Pantsar (2014) seems to be able to explain two of the three main characteristics of the traditional image of arithmetical knowledge. Although arithmetical knowledge is not strictly a priori, it is contextually so. Moreover, this context is set by biological primitives, which explains why arithmetical truths appear to be objective to us. For someone dealing with arithmetic - from simple calculations to proving theorems - it is quite understandable to get the image she is engaging in a priori pursuit of objective truths. For all practical purposes, she is doing just that - in a context that she is thoroughly familiar with from years of experience in processing observations in terms of numerosities.

\section{Necessity}

While objectivity and the a priori appearance of arithmetic seem be to explained in sufficiently strong forms in the contextual a priori account, it is not clear that the impression that arithmetical knowledge is necessary can be explained as easily. In the tradition of analytic philosophy, a priori and necessary truths have often been thought to amount to the same thing, that is, they are grounded only in the meaning of the terms involved. ${ }^{6}$ Clearly such a connection picks out an important class of sentences, those of the type "all bachelors are unmarried". 7 But I have argued that arithmetical statements do not belong to that class, that is, they are not strictly a priori. In the traditional account of conflating a priori and necessary knowledge, it would seem to follow that arithmetical truths cannot be necessary, either.

When discussing modal concepts, arithmetical statements like " $2+1=3$ " are often given as standard examples of necessary truths - that is how obvious the necessity of arithmetical truths is generally taken to be in philosophy. But if we understand necessary truths - as is usually done since Kripke (1963) and Lewis (1970), among others - as true in all possible worlds, arithmetical truths according to the contextual a priori account would not be necessary. There are possible worlds in which human beings and other animals with protoarithmetical ability did not develop. If arithmetical truths are based on the proto-arithmetical ability, it would appear to follow that they are not true in

\footnotetext{
${ }^{6}$ Kripke (1980, p. 35) states that in contemporary (meaning around the year 1970) discussion very few philosophers even made the Kantian distinction between a priori and necessary.

${ }^{7}$ Here I am ignoring what I see as trivial empirical elements, like the fact that we need to see or hear (or feel) an explanation of the words involved in order to see the truth of sentences like "all bachelors are unmarried".
} 
such possible worlds. That is a quite troubling prospect. Most of us would not be ready to accept that " $2+1=3$ " could be false, yet that would seem to be a possibility if we accept the contextual a priori account.

Are there ways out of this problem? One potential solution could be to think of arithmetical knowledge as arising from the proto-arithmetical ability, but arithmetical statements being true regardless of there being agents with knowledge of them. This way we could avoid, for example, the uncomfortable position that " $2+1=3$ " could cease to be an arithmetical truth if there no longer were sufficiently developed biological organisms. But what would be the ontological status of arithmetical truths in such a scenario? Any answer would seem to come worryingly close to platonism or some other position that takes arithmetical truths to be totally mind-independent, which puts the whole point of the contextual a priori theory in question. One of the main strengths of the theory is its lack of ontologically problematic assumptions, in particular that of a mind-independent reality of mathematical objects. While certainly not contradictory with the contextual a priori, assuming a mind-independent existence of arithmetical truths at the very least goes against the general spirit of the theory.

Another possibility could be to accept that " $2+1=3$ " may not be a truth in all possible worlds, but insist that this does not imply that it could be false. It could be the case that even primitive forms of arithmetic never emerged perhaps because no sufficiently developed biological organisms developed - but if it did, it would have been the case that " $2+1=3$ " is true. In such a scenario, either " $2+1=3$ " is true, or there are no arithmetical truths. But also this solution seems to make an ontological claim that is too strong for the general spirit of the contextual a priori theory. According to the theory, arithmetic is based on our cognitive architecture. But it is possible that the brain structure could evolve in a sufficiently different manner to give birth to a radically different kind of arithmetic. To think that arithmetical knowledge must develop among the lines it has in our actual world suggests a stronger ontological status for arithmetic - that arithmetical truths somehow determine the way biological organisms must develop.

\section{What are arithmetical truths?}

Based on the considerations above, explaining the apparent necessity of arithmetical truths appears to be a difficult task for the contextual a priori theory. The main obstacle seems to be combining an account that takes arithmetical knowledge to be based on biological primitives with the position that arithmetical truths must be true also in possible worlds where no such biological organisms exist. The big question is, however, how much weight we should give to such possible worlds. What if we instead limited our focus only to possible worlds with sufficiently developed biological organisms? ${ }^{8}$

\footnotetext{
${ }^{8}$ By "sufficiently developed", I mean sufficiently developed along the lines that evolution took in our actual world. I do not wish to engage here in speculation about highly developed
} 
Such a move may sound unwarranted, but I claim that it is because in philosophy we are still too committed to the traditional conception of arithmetic as a priori necessary truths. We use " $2+1=3$ " as an example of a necessary truth because it is thought to give a prime example of a statement that is true regardless of the characteristics that possible worlds may have. But inherent in that is a strong form of objectivism about arithmetical truths. It may be easy to accept, for example, the position that arithmetical truths are eternal. However, it is not as easy to give that position a satisfactory explanation without evoking strong ontological commitments.

Perhaps we need to have a platonist conception that arithmetical truths concern mind-independent mathematical objects, which is hardly a satisfactory explanation for the contextual a priori theory. Alternatively, we may believe that arithmetical truths are actually logical truths. Yablo (2002) has argued for the position that arithmetic arises from our cognitive mechanisms, but that arithmetical facts are actually facts of first-order logic. Since logical truths are commonly accepted as really being necessary, this explains why arithmetical truths appear to be necessary.

However, it is not obvious how moving the focus to logic adds explanatory value. First of all, while we certainly can present finite quantifier-free arithmetical truths like " $2+1=3$ " as truths of first-order logic, there is no generally accepted way of deriving full arithmetic purely from logic. ${ }^{9}$ But even if we could derive full arithmetic from logic, would it explain anything further? Yablo argues that logical truths are indeed necessary since they are tautologies. It is not clear, however, why logical truths should be somehow easier to accept as necessary than arithmetical truths. Indeed, the necessity of " $1+1=2$ " seems to be just as obvious as that of logical tautologies. Yablo explains this by claiming that arithmetical truths are in fact logical truths, and thus equally necessary. An alternative explanation would be that both simple arithmetical and simple logical truths are enforced upon us by our cognitive architecture, thus making both contextually a priori in character. Without further arguments, it is hard to see how we can distinguish between the apparently different natures of logical and arithmetical truths.

One such further argument has come from Maddy (2014), who also holds that simple arithmetical statements are shorthand for logical truths. Her account differs from Yablo's in that she allows that we need something more for the "..." part in the progression of natural numbers, i.e. when moving from finite numbers to the infinity of them. For this, she argues - following Bloom (2000) - that:

Much as our primitive cognitive architecture, designed to detect [the logical structure of the world], produces our firm conviction in sim-

\footnotetext{
biological organisms with completely different cognitive systems.

${ }^{9}$ The neo-Fregeanism of Wright (1983) comes perhaps closest to fulfilling the logicist idea but in addition to using second-order logic, which is sometimes seen as going against the logicist ideal, he uses a non-logical axiom - the so-called Hume's principle - in deriving the axioms of arithmetic. Yablo's own solution is to interpret universal quantifiers in arithmetic as infinite chains of conjunctions.
} 
ple cases of rudimentary logic, our human language-learning device produces a comparably unwavering confidence in this potentially infinite pattern. (Maddy 2014, p. 234)

Thus Maddy's theory is similar to the contextually a priori account when it comes the move beyond the primitive origins. However, when it comes to those primitive origins, her account has two differences. First of all, she holds that the cognitive architecture is about logical structure, i.e. it is proto-logical rather than proto-arithmetical. At this point, it is impossible to commit strongly to either position based on the empirical data. However, for the present matter at hand, it seems irrelevant whether our primitive abilities are primarily logical or arithmetical - or perhaps equally both. If they shape our experiences and thus constrain the content of arithmetic, the contextual a priori is equally applicable to both explanations.

The second difference is more relevant. In the above quotation, Maddy writes that our primitive cognitive architecture detects the logical structure of the world. In the contextual a priori account, the cognitive abilities are only thought to categorize our observations in terms of numerosities. This is clearly an important difference, since for Maddy rudimentary arithmetic (arithmetic without the "..." part) comes from the structure of the world, thus proposing stronger objectivism than the contextual a priori account.

This comes down to a version of the old Kantian problem of distinguishing between objective features of the world and those imposed by our cognitive architecture. Are we designed to detect the logical structure of the world or does our cognitive architecture impose that structure on our observations? Unfortunately, here I cannot go deeper into such considerations. But ontological carefulness being at the heart of the contextual a priori project, I am not prepared to accept Maddy's strong objectivist claim. In any case, her position is in no way incompatible with the contextual a priori account. Perhaps arithmetic is ultimately about objective features of the world and as such arithmetical truths are mind-independent and necessary in a stronger sense. What I am arguing for here is that even without making that assumption - for which I do not see enough grounds - we can have a perfectly satisfactory account of the apparent necessity of arithmetical truths.

This is an important point, because it seems that augmenting the contextual a priori theory of arithmetical knowledge with a strong form of objectivism would take away power from my argumentation. Even if the theory were essentially correct, we would now need to ask how the biological primitives are connected to either the platonic world of mathematical objects or the logical structure that all possible worlds share - which are just the kind of problems that the contextual a priori account was supposed to avoid.

\section{$5 \quad$ Necessary contextual a priori knowledge}

It seems to be the case, however, that the reason arithmetical truths are such popular examples of necessary truths is not simply because they appear to be 
true in all possible worlds. Instead, I claim that that impression is based on another impression: that arithmetical truths are a priori. If we can find out a truth simply by reflection, it is easy to accept that the truth in question holds in all possible worlds. But of course I argue that we do not find out arithmetical truths purely a priori. Consequently, we should also be prepared to reconsider our understanding of arithmetical necessity.

In modern analytic philosophy it is widely accepted that Kripke in his Naming and Necessity (1980) demolished the old notion that only a priori knowledge can be necessary. Most importantly, he argued that there are cases of necessary a posteriori knowledge. The best-known example of this is the sentence "Hesperus is Phosphorus". Both "Hesperus" and "Phosphorus" are proper names and as such pick out the same thing in every possible world where that thing exists. For "Hesperus", the thing is the evening star, and for "Phosphorus", the morning star. But we know that "Hesperus" and "Phosphorus" actually refer to the same thing, the planet Venus. So the sentence "Hesperus is Phosphorus" is necessarily true: in all possible worlds "Hesperus" and "Phosphorus" refer to the same thing. Since the fact that both refer to the planet Venus was discovered empirically, Kripke argues, "Hesperus is Phosphorus" is a case of necessary a posteriori knowledge.

Could we use Kripke's strategy above to save the necessity of arithmetical knowledge in the contextual a priori account? At first sight, the argument looks promising, since the concept of necessity is expanded beyond strictly a priori truths - which arithmetical truths are not, according to my account. The key point in Kripke's argument, however, is by no means guaranteed to apply to the case at hand. Kripke introduced the term "rigid designator" for terms that pick the same object in all possible worlds in which that object exists. In this he relied on a causal theory of reference. We can know that the term "Hesperus" picks out the evening star in every possible world where the evening star exists because there is causal link from the name "Hesperus" to the event of giving it its reference, the planet Venus.

But in the case of arithmetical knowledge, is there a causal link between, say, the numeral "two" and its reference? Can we think of numerals as rigid designators? Kripke himself was notoriously reluctant to discuss the finer details (e.g., the nonexistence of objects) of rigid designation. Among the distinctions he explicitly ignored was that of de jure rigidity and de facto rigidity (Kripke 1980, p. 21 n. 21). De jure rigidity is designation by stipulation, like in the case of Hesperus and Phosphorus, whereas de facto rigidity can be designation by description. In Kripke's example, "the smallest prime" is a de facto rigid designator because it picks out a unique object, the number two.

De facto rigid designation would seem to open up interesting possibilities. Let us continue with the contextual a priori account and deny that arithmetical knowledge comes from some kind of direct access to mind-independent natural numbers. In that case, whether or not there are such things as natural numbers, there could hardly be de jure designation for numerals. However, it appears that also in this case descriptions like "the smallest prime" do designate rigidly. Whenever we $d o$ have natural numbers, "the smallest prime" picks out the same 
number.

When it comes to natural numbers, the question of existence is obviously more tricky than with the planet Venus. The whole point of approaches like Pantsar (2014) is that we do not need to evoke mind-independent existence for numbers in order to have feasible epistemology of arithmetic. But at the same time, the theory is based on biological primitives and as such entails a high level of objectivity for arithmetical truths. There may not be such an object as the natural number two, but there is extremely strong objectivity in the way we use the numeral "two". 10

However, it seems that the latter type of objectivity is enough for a Kripketype theory of reference. " $2+1=3$ " is true in all possible worlds where enough arithmetical or proto-arithmetical ability has developed to give reference for the numerals "2", "1" and " 3 ". It is not necessary that there is a single object, the natural number two, to which we refer to when we use the numeral "two". Rather, "two" can designate different things at different levels. When a child first learns to use the numeral " $\mathrm{two}$ " correctly, her understanding of arithmetic is very limited and the numeral refers to her proto-arithmetical knowledge arising from seeing (or hearing or touching) two things.

When arithmetic develops beyond this primitive level, we find explicit ways of characterizing what "two" refers to, for example, by using the successor operation twice on zero. When we refer to a number like eleven, in which case our proto-arithmetical ability no longer suffices, we refer to using the successor operator on zero eleven times. That is how we learn to count and do basic arithmetic as children. When we use the numeral "two" in this more developed context, we are referring to something conceptually different from what the child does with her proto-arithmetical use of "two".

At a first glance, it would then seem obvious then that "two" cannot be a rigid designator in either the de jure or the de facto sense, as it can refer to different things. However, this is not so simple. It is clear that if we do not include arithmetical objects in our ontology, in the strict sense numerals cannot be rigid designators. But while in the contextual a priori account numbers are not postulated to exist, numerals are still thought to refer to something strongly objective, i.e., the concepts developed in arithmetic which are constrained by our biological structure. There is no single object that the numeral "two" designates, but it still manages to pick out something that is at the basis of animal and infant behaviour, as well as of our developed arithmetic. If arithmetic is constrained by biological primitives in a strong way as argued in the contextual a priori account, the numeral "two" picks out that something in all possible worlds where sufficiently developed biological organisms develop.

This is not quite the rigid designation of Kripke, but it is essentially similar - as long as we turn the focus on cognitive abilities instead of objects. Rather than talk about all the worlds where the object exists, we focus on all the

\footnotetext{
${ }^{10}$ It should be noted that Kripke also introduces (1980, p. 28) the term "strong rigid designation" for terms referring to necessary existents, presumably including numbers. It is unclear to me whether there are conceivable cases of strong rigid designation, but it should be clear by now that natural numbers should not be considered to be such.
} 
worlds where sufficiently developed biological organisms develop. Rather than talk about a single object, we focus on an ability with quantities that has a continuous development from the proto-arithmetical ability to developed formal arithmetic. With these adjustments, the use of the numeral "two" has essentially the same characteristic as Kripke's rigid designation: namely, it picks out the same thing in all the possible worlds where that thing exists.

What that "thing" is in case of arithmetic, however, is more complicated than in the case of Venus. At the stage of our development when we understand abstract objects, we can conveniently refer to the abstract natural number two. That is why the numeral "two" appears to be a rigid designator. However, under further analysis it is revealed that "two" refers to something much more complicated, a development which has different stages starting from the protoarithmetical origins and ending up with formal axiomatic arithmetic. But importantly, that development is constrained by our cognitive architecture and thus guarantees a strong form of objectivity for the reference of "two". While this is not Kripke's rigid designation, it is rigid enough for our current purposes - explaining the apparent necessity of arithmetical knowledge.

Whatever the exact philosophical characteristics of natural numbers may be, our shared biological structure and the arithmetical knowledge built on that will make sure that the numeral "eleven" will pick out the same "object" from what we at the furthest stage of the development describe as the abstract domain of arithmetic, the set of natural numbers. But there is no need to postulate that such a set or its elements actually exist. Perhaps they do, but we can also explain arithmetical knowledge and the reference of numerals without evoking such platonic properties.

There are, of course, possible worlds in which arithmetic did not develop. But in Kripke's theory it is enough that the name picks out the same thing in all the possible worlds where the thing exists. When we adjust our understanding of arithmetical objects as above, a Kripke-like theory fits well with my theory of contextual a priori epistemology of arithmetic. It is enough that a numeral picks out the same thing in all the possible worlds where sufficiently developed biological organisms develop.

Kripke argued that knowledge can be necessary and a posteriori. If that is the case, it follows that arithmetical knowledge can be both necessary and contextually a priori. I hope to have shown that in a modified Kripkean framework we can explain at least the apparent necessity of arithmetical truths. Perhaps arithmetical truths are necessary also in the strong sense of Kripke, i.e. true in all possible worlds. The contextual a priori account does not take a stand on that question. But it can explain why arithmetical truths are true in all possible worlds with sufficiently developed biological organisms. For the purposes of this paper, that is enough. We have explained, based on the contextual a priori account of the epistemology of arithmetic, the last of the three apparent characteristics that arithmetical knowledge has. In addition to appearing to be both a priori and objective, it has now been shown why arithmetical knowledge 
appears to be necessary. ${ }^{11}$

\section{References}

Bloom, P. (2000). How Children Learn the Meanings of Words. Cambridge, MA: MIT Press.

Carey, S. \& Sarnecka, B.W. (2006). "The development of human conceptual representations", in M. Johnson \& Y. Munakata (Eds.), Processes of Change in Brain and Cognitive Development: Attention and Performance XXI, pp. 473496.

Dehaene, S. (2011). Number Sense, Second Edition, New York: Oxford University Press.

Dehaene, S. \& Brannon, E. (eds.) (2011). Space, Time and Number in the Brain, London: Academic Press.

Field, H. (1980). Science without Numbers: A Defense of Nominalism, Princeton: University Press.

Frege, G. (1879). Begriffsschrift. Halle.

Frege, G. (1884). The Foundations of Arithmetic, translated by J. L. Austin. Oxford: Basil Blackwell 1974.

Jenkins, C. (2008). Grounding Concepts, Oxford: Oxford University Press.

Kant, I. (1781). Critique of Pure Reason, translated/edited by P. Guyer and A. Wood, Cambridge: Cambridge University Press, 1997.

Kitcher, P. (1983). The nature of mathematical knowledge. New York: Oxford University Press.

Kripke, S. (1963). Semantical Considerations on Modal Logic. Acta Philosophica Fennica, 16: 83-94.

Kripke, S. (1980). Naming and necessity, Cambridge, MA: Harvard University Press.

Kuhn, T. (1993). Afterwords, in Paul Horwich (ed.), World Changes, Cambridge, MA: MIT Press: 331-332.

Lakoff, G. \& Núñez, R. (2000). Where Mathematics Comes From, New York: Basic Books.

Lewis, D. (1970). General Semantics. Synthese, 22: 18-67.

Maddy, P. (2014). A Second philosophy of arithmetic, The Review of Symbolic Logic, Vol. 7: 222-249.

\footnotetext{
${ }^{11}$ This research was funded by the Academy of Finland, whose support is acknowledged with great gratitude. A big thank you is in place to the participants of the FilMat conference Philosophy of mathematics: objectivity, cognition, and proof in Milan. The discussions there helped formulate the arguments of this paper. The final version of this paper was written during a visit to the University of California, Irvine. I am grateful for the discussions with the staff there, especially with Sean Walsh, Penelope Maddy and Kai Wehmeier. Finally, with gratitude I note that the paper as it appears here benefitted greatly from the thorough referee reports of two anonymous referees.
} 
Nieder, A. (2011). The Neural Code for Number, in Dehaene, S. \& Brannon, E. (eds.): Space, Time and Number in the Brain, London: Academic Press 2011: 107-22.

Pantsar, M. (2014). An empirically feasible approach to the epistemology of arithmetic. Synthese, 191, 4201-4229.

Putnam, H. (1976). Two Dogmas' Revisited," in Realism and Reason: Philosophical Papers: Volume 3 Cambridge: University Press, 1983: 87-97.

Spelke, E. (2011). "Natural Number and Natural Geometry", in Dehaene \& Brannon (eds.): Space, Time and Number in the Brain, London: Academic Press 2011: pp. 287-318.

Wittgenstein, L. (1956). Remarks on the Foundations of Mathematics, Revised Edition, von Wright G.H., Rhees, R. and Anscombe, G.E.M. (eds.), translated by Anscombe, G.E.M. Oxford: Basil Blackwell 1978.

Wright, C. (1983). Frege's Conception of Numbers as Objects, Aberdeen: Aberdeen University Press.

Wynn, K. (1992). Addition and subtraction by human infants, Nature, 358, 749-751.

Yablo, S. (2002), Abstract Objects: A Case Study. Noûs, 36: 220-240. 